\title{
GABA-induced inactivation of Cebus apella V2 neurons: effects on orientation tuning and direction selectivity
}

\author{
A.K. Jansen-Amorim ${ }^{1}$, M. Fiorani ${ }^{2}$ and R. Gattass ${ }^{2}$ \\ ${ }^{1}$ Instituto de Ciências Biológicas, Universidade Federal do Pará, Belém, PA, Brasil \\ ${ }^{2}$ Instituto de Biofísica Carlos Chagas Filho, Universidade Federal do Rio de Janeiro, Rio de Janeiro, RJ, Brasil
}

\begin{abstract}
We investigated the GABA-induced inactivation of V2 neurons and terminals on the receptive field properties of this area in an anesthetized and paralyzed Cebus apella monkey. Extracellular single-unit activity was recorded using tungsten microelectrodes in a monkey before and after pressure-injection of a 0.25 or $0.5 \mathrm{M}$ GABA solution. The visual stimulus consisted of a bar moving in 8 possible directions. In total, $24 \mathrm{~V} 2$ neurons were studied before and after blocker injections in 4 experimental sessions following GABA injection into area V2. A group of 10 neurons were studied over a short period. An additional 6 neurons were investigated over a long period after the GABA injection. A third group of 8 neurons were studied over a very long period. Overall, these 24 neurons displayed an early (1-20 min) significant general decrease in excitability with concomitant changes in orientation or direction selectivity. GABA inactivation in area V2 produced robust inhibition in $80 \%$ and a significant change in directional selectivity in $60 \%$ of the neurons examined. These GABA projections are capable of modulating not only levels of spontaneous and driven activity of $\mathrm{V} 2$ neurons but also receptive field properties such as direction selectivity.
\end{abstract}

Key words: Visual cortex; Single units; Extracellular recordings; New World monkeys

\section{Introduction}

Since the early works of Hubel and Wiesel (1) on the response properties of $\mathrm{V} 1$ neurons, a substantial amount of data on the functional organization of the visual cortex has been accumulated. In each visual cortical area, neurons have a unique inventory of receptive field properties. Each of these properties is combined with those of neighboring neurons to form orderly representations of stimulus features; these different representations are superimposed within the same population of neurons (1). Since the discovery that cells in the visual cortex are selective for the orientation of contours, the mechanisms underlying cortical orientation selectivity have been the subject of intense debate. These features are widely believed to emerge from particular combinations of ascending, descending and lateral projections $(2,3)$. The major excitatory input to cells in all layers is derived from a massive convergent input from other cortical cells. This cortical input has the potential to enhance orientation and direction selectivity via excitatory interactions both within the same orientation column (4) and between columns with like orientation preferences (5).
Neurons in early visual areas, such as V1 and V2, are selective for the orientation and direction of movement of visual stimuli. It has been suggested that some receptive field properties of cortical neurons, such as orientation and direction selectivity, may be attributed to the inhibitory influence of intrinsic circuits on incoming information $(6,7)$. The inactivation of intrinsic inhibitory processes impairs both orientation and direction selectivity $(8,9)$. The recording of neurons targeted and inactivated by gama-amino butyric acid (GABA) at sites in cat area 18 revealed that inactivation at sites with similar orientation preference produced a significantly greater effect on directionality relative to the minor effect on directionality observed following inactivation of regions of cross-orientation. Effects on orientation tuning were produced almost exclusively by cross-orientation sites, while changes in directionality were produced mainly by sites of the same orientation $(10,11)$. Studies that used labeling with tritiated nipecotic acid to identify GABAergic neurons at the site of inactivation showed that orientation-tuning V2 neurons change their specificity due to the inhibition of

Correspondence: R. Gattass, Instituto de Biofísica Carlos Chagas Filho, Universidade Federal do Rio de Janeiro, 21941-900 Rio de Janeiro, RJ, Brasil. E-mail: rgattass@gmail.com

Received December 8, 2012. Accepted May 9, 2013. First published online July 22, 2013. 
cross-orientation-selective neurons via GABAergic neurons (7). In conclusion, in the primary (V1) and secondary (V2) visual areas of monkeys and cats, the orientation and direction selectivity of neurons depend on the inhibitory influence of basket cells projecting to orientation- and direction-selective functional modules $(6,7,11-13)$.

In the present study, we investigated whether extrinsic GABA-mediated projections (feed-forward or feedback) and/or intrinsic projections from area V2 directly interfered with the orientation and direction selectivity of $\mathrm{V} 2$ neurons. $V 2$ has a surface area of about $625 \mathrm{~mm}^{2}$. The injections of GABA encompassed $4-10 \mathrm{~mm}^{2}$ of the cortical area (or a volume of $8-20 \mathrm{~mm}^{3}$ ), representing up to $1.6 \%$ of V2 (14). The injections of lidocaine encompassed about $36 \mathrm{~mm}^{2}$ $\left(96 \mathrm{~mm}^{3}\right)$, representing up to $6.8 \%$ of $\mathrm{V} 2$, and almost its entire width of $6-7 \mathrm{~mm}$. These estimates are consistent with the research of Hupé et al. (15). They performed a quantitative study on the spread of GABA after pressure injections into the cortex and predicted that the injected volume would spread throughout the extracellular space, occupying an ellipsoid shape. Considering the extent of area V2 determined by Rosa et al. (14), we extended the predictions of Hupé et al. (15) regarding the relationship of injected volume and occupied extracellular volume. We predicted that GABA injection volumes between 120 and $600 \mathrm{~nL}$ would inactivate $0.02-0.16 \%$ of area $\mathrm{V} 2$, while 1.8 and $6 \mu \mathrm{L}$ lidocaine would inactivate $0.2-1.6 \%$ of $\mathrm{V} 2$.

We studied the receptive field properties of $\mathrm{V} 2$ neurons before and after the inactivation of a small portion of area V2 in the capuchin monkey (Cebus apella). Several aspects of the visual system of this New World monkey, including the thalamic organization (16-18), intrinsic circuitry of V1 (19-21), and topographical characteristics of areas V1, V2, MT, and V4 $(14,22-25)$ have been studied for almost two decades, making this monkey a suitable experimental model for this study. In addition, we recently studied the effect of GABA inactivation of visual area MT (26), visual area V4 (27), and previously (2004) the pulvinar (28) on the direction and orientation selectivity of V2 neurons. These studies enabled us to make direct comparisons regarding GABA inactivation on the feedback connections from MT and V4.

\section{Material and Methods}

\section{Animals}

One adult male Cebus apella monkey was used in 5 recording sessions, performed once a week. All the experimental protocols were conducted following the National Institutes of Health $(\mathrm{NIH})$ guidelines for animal research and were approved by the Committee for Animal Care and Use of the Instituto de Biofísica Carlos Chagas Filho, Universidade Federal do Rio de Janeiro (2011).

\section{Location of $\mathrm{V} 2$ and preparations for the recording sessions}

$\mathrm{V} 2$ is a belt of cortex located in the opercular region of the occipital pole in front of $\mathrm{V} 1$. It is limited posteriorly by $\mathrm{V} 1$, except in the most anterior region of the calcarine sulcus, which borders the area of prostriata of Sanides $(14,21)$. V2 is limited anteriorly by V3 except at the representation of the fovea region at the lateral surface between the lunate sulcus and the inferior occipital sulcus, where V2 borders V4 (21). In C. apella, V2 is wider $(8-11 \mathrm{~mm})$ in the region of intermediate representation of the visual field (about $10^{\circ}$ eccentricity) and narrows (4-5 $\mathrm{mm}$ ) in the bottom of the sulci representing the foveal region (14).

Prior to the recording sessions, and under anesthesia and aseptic conditions, a head bolt and a recording chamber were attached to the skull. The position of the recording chamber was determined by visualizing the vascularization of the sulci through the skull. The superior temporal sulcus (STS) is always visible and it constitutes an important anatomical landmark. The intraparietal sulcus is also quite visible, but it is less conspicuous than the STS. The lunate sulcus is far less clear than the STS and the intraparietal sulcus. The location of the posterior border of $\mathrm{V} 2$ is $2-3 \mathrm{~mm}$ posterior to the lunate sulcus. Using stereotaxic coordinates and the position of the cortical sulci, we positioned the recording chamber such that we had access to area V2. During each recording session, the animal was anesthetized with a $5 \%$ ketamine hydrochloride solution (30 mg/kg, intramuscular; Ketalar, Parke Davis, Brazil) and maintained with a mixture of $70 \%$ nitrous oxide and $30 \%$ oxygen combined with a continuous intravenous infusion of fentanyl citrate $\left(0.003 \mathrm{mg} \cdot \mathrm{kg}^{-1} \cdot \mathrm{h}^{-1}\right)$. After endotracheal intubation with a newborn \#3.5 cannula, the monkey was also immobilized with pancuronium bromide $\left(0.1 \mathrm{mg} \cdot \mathrm{kg}^{-1} \cdot \mathrm{h}^{-1}\right)$ and maintained under mechanical ventilation (Primate ventilation pump, Harvard Apparatus, USA) adjusted to obtain 3-4\% expired $\mathrm{CO}_{2}$. Electrocardiogram readings, body temperature, and end-tidal $\mathrm{CO}_{2}$ were monitored continuously. Post-surgical analgesia was maintained for 3 days with the administration of fentanyl skin patches (Durogesic ${ }^{\mathbb{R}}$, Janssen-Cilag, Brazil).

After implant of the recording chamber, the bone inside the chamber was removed, leaving the dura intact. After 10 days, a thick fibrous growth covered the dura and prevented the drying of the cortex.

\section{Recording sessions}

To locate V2, we penetrated the cortex with $1 \mathrm{M} \Omega$ impedance tungsten microelectrodes (Frederik Haer 250 $\mu-\phi$, USA), using stereotaxic coordinates and sulcal landmarks $(14,23)$. The V2 stereotaxic coordinates were used to allow access to the central lower field representation of area V2 in subsequent recording sessions. The injection and recording sites were typically located $6-10 \mathrm{~mm}$ from the midline, $1 \mathrm{~mm}$ behind the lunate sulcus, at the depth of 4-6 mm from the cortical surface.

\section{Inactivation devices}

The injection system used for inactivation consisted of 
two stainless steel tubes (external diameter: $400 \mu \mathrm{m}$; internal diameter: $200 \mu \mathrm{m}$ ) mounted around a tungsten microelectrode. The entire system measured approximately $800 \mu \mathrm{m}$. A pneumatic pico pump (Model PV 820, World Precision Instrument, USA) delivered the GABA (Sigma, USA) solution to the two external stainless steel tubes through a polyethylene connection. GABA was diluted in deionized water and stored at $4{ }^{\circ} \mathrm{C}$. Lidocaine was used straight from the bottle. With this system, it was possible to deliver 120 to $600 \mathrm{~nL}$ of a $0.25 \mathrm{M}$ GABA solution or 1.8 to $6 \mu \mathrm{L} 2 \%$ lidocaine (Xylocaine ${ }^{\mathbb{R}}$, Astra Zeneca, USA) to the region of interest. The injections were administered over a period of $30 \mathrm{~s}$.

After the topographical site was localized in the V2, a single tungsten microelectrode was replaced by the injection system, with stainless steel tubes placed approximately $800 \mu \mathrm{m}$ apart, to inactivate V2 neurons. Single-unit activity from area $\mathrm{V} 2$ was recorded using tungsten microelectrodes. The activity was amplified (10,000X) and filtered (band pass, $60-6000 \mathrm{~Hz}$ ), and single spikes were sampled at $166 \mathrm{kHz}$ by a waveform discriminator system (SPS-8701, Signal Processing System, Australia). The computer-based discriminator SPS-8701 uses a template-matching algorithm to sort up to three spikes. Extracellular single-unit spike events were stored using CORTEX software (Laboratory of Neuropsychology, $\mathrm{NIMH} / \mathrm{NIH}$, USA) for offline analysis. The receptive fields were initially localized and mapped using a hand-plot mapping procedure.

The receptive field automatic mapping procedure was based on computing the latency-corrected neuronal activity in response to elongated bars moving in 1 of 8 directions of motion. Initially, peristimulus time histograms (PSTHs) were computed based on 10 stimulus presentations, using a bin width of $10 \mathrm{~ms}$. Single-trial spike trains were used to produce PSTHs that were aligned to stimulus onset. The PSTHs were then smoothed using a normal convolution filter with a 200-ms time window, which resulted in the time spike density function. This continuous and derivable function well characterizes the neuronal firing pattern (see Ref. 26).

\section{Inactivation paradigm}

Area V2 was inactivated until a significant reduction of the recorded activity at the injection site was obtained. Data were collected immediately before and after the injection, and several blocks of recording protocols were acquired until recovery of V2 cellular activity. The recording sessions typically continued for $14 \mathrm{~h}$. The interval between experimental sessions was at least 10 days. With this paradigm we could not discriminate whether extrinsic GABA-mediated projections (feedforward or feedback) or intrinsic projections from area V2 were interfering with the orientation or direction selectivity of V2 neurons. We therefore added very large injections of a local anesthetic to the paradigm to try to segregate the effect on the responsiveness of the neuron from that on the circuit underlying directional or orientation selectivity. For these reasons we used very large injections of $2 \%$ lidocaine $(1.8-6 \mu \mathrm{L})$ to compare to restricted (120-600 $\mathrm{nL}$ ) injections of $0.25 \mathrm{M} \mathrm{GABA}$.

Recordings from area V2 were obtained before (control) and during several sequential time blocks at different time points (1-300 min) after GABA inactivation of area $V 2$. The neuronal responses at the injection site in area V2 were analyzed by comparing an effective direction-selective polar plot (polargram) with a poststimulus raster in a histogram plotted as the spike density functions of the neuronal discharge. These effects were compared to the inactivation with the local anesthetic lidocaine.

The electrodes were used once and discarded. The inactivation system was sterilized before use with neutral detergent, bleach, and Germi-Rio (Rioquímica, Brazil).

\section{Visual stimulation}

Prior to the recording sessions, gas-permeable contact lenses were used to focus the monkey's eyes on a computer monitor placed $57 \mathrm{~cm}$ in front of the animal. The positions of the blind spot and fovea were plotted onto the computer screen with a reversible ophthalmoscope. The stimulus consisted of a thin white bar $\left(18 \times 0.5^{\circ}\right)$ that appeared in four random orientations $\left(0^{\circ}, 45^{\circ}, 90^{\circ}\right.$, and $135^{\circ}$ ), crossed the screen in a direction perpendicular to its orientation at a velocity of $10^{\circ} / \mathrm{s}$, and passed through the receptive fields of all recorded neurons. We continuously tested the direction of motion selectivity before and after GABA injection. Note that we did not segregate orientation selectivity from axis-of-movement selectivity. Therefore, the moving bar in this configuration was used to determine both the direction and axis of movement selectivity.

\section{Data analysis}

The changes in V2 neuron direction selectivity before and immediately after GABA injection into area V2 were evaluated using analysis of variance (ANOVA) (MATLAB toolbox, MathWorks Inc., USA). To determine the significance of changes in the directionality of V2 neurons in response to visual stimulation, data from each stimulus direction were submitted to one-way ANOVA (1WA) followed by a post hoc least significant difference test. To determine the statistical significance of the effects on V2 neuron direction selectivity before and after GABA injection into area V2, cell activity under each condition was analyzed using two-way ANOVA (2WA). We also performed a statistical evaluation of the recovery after GABA injection by evaluating cell activity in the control condition, before GABA injection and after the GABA-induced effects had vanished, using 2WA. Finally, some cells that did not exhibit a statistically significant directional component instead showed a significant bidirectional response. By 
grouping the conditions with the same stimulus orientation moving in opposite directions, we tested this bidirectional component. The four groups of data generated by this procedure were then submitted to 1WA to evaluate changes in the selectivity of the cell for axis of movement. Probability values mentioned in the text, which were related to the changes in direction selectivity, were evaluated by ANOVA unless otherwise specified.

The selectivity of the neurons was examined with a standard test of circular tuning to determine the magnitude of the GABA-induced changes in both direction and orientation selectivity across the population. The orientation selectivity index (OI) was calculated for each neuron according to the following formula, as proposed by Sato et al. (6): Ol $=\left[\left(\Sigma \mathrm{R}_{\mathrm{i}} \sin \left(2 O_{\mathrm{i}}\right)\right)^{2}+\left(\Sigma\left[\mathrm{R}_{\mathrm{i}} \cos \left(2 O_{\mathrm{i}}\right)\right)^{2}\right]^{0.5} / \Sigma \mathrm{R}_{\mathrm{i}}\right.$, where $R_{i}$ represents the magnitude of response to each stimulus orientation, $\mathrm{O}_{\mathrm{i}}$. The rate of spontaneous activity, which was measured for the $700 \mathrm{~ms}$ window before stimulus presentation, was subtracted from the stimulusdriven responses. The response to each stimulus orientation is expressed as a vector with direction $20_{\mathrm{i}}$ and length $R_{\mathrm{i}}$. This index varied between 1 and 0 , with an OI value of 1 indicating complete selectivity to a particular orientation and a value of 0 indicating equal responsiveness to all orientations.

Direction selectivity indices (DI) were also quantified by the directional index, as described by Wang et al. (29). An index of 1 indicates sharply tuned cells, whereas an index of 0 indicates non-tuned cells. We used a paired Student $t$-test to evaluate changes in the mean OI and DI in the cell population. Using this test, we also evaluated the recovery time after GABA injection by comparing cell activity in the control condition both before the GABA injection and after the GABA-induced effects had vanished. In addition, we used the individual index values to reveal any bias in the population. We considered a change greater than 0.20 in the OI or DI to be significant. Population bias was evaluated using the $\chi^{2}$ test.

\section{Histological procedures}

After a complete set of inactivation sessions, the animal was killed for histological processing to localize the injection and recording sites and to examine the structure of the cortical layers. At the end of the last recording session, the animal was deeply anesthetized with sodium pentobarbitone $(30 \mathrm{mg} / \mathrm{kg})$ and perfused intracardially with saline, followed by $2 \%$ paraformaldehyde in phosphatebuffered saline (PBS), 2\% paraformaldehyde in PBS supplemented with $2.5 \%$ glycerol, PBS supplemented with $5 \%$ glycerol, and PBS supplemented with $10 \%$ glycerol. Frozen sections (70 $\mu \mathrm{m}$ thick) were cut on a cryostat and mounted on glass slides or kept in PBS at $4^{\circ} \mathrm{C}$. The sections were stained using the Nissl (cresyl violet) stain and were analyzed on a slide projector and microscope to determine the locations of the electrode tracks and the absence of cortical lesions.

\section{Results}

GABA inactivation of visual area V2 produced a general decrease in the excitability of the neurons, which included a decrease in spontaneous and driven activities followed by changes in direction and/or orientation selectivity.

The results of the V2 inactivation in the same area of the recordings were combined into three groups. The first group, composed of 6 neurons, was subjected to 1 injection of $120 \mathrm{~nL} 0.5 \mathrm{M}$ GABA. The second group, composed of 8 neurons, was subjected to 2 injections ( 1.8 and $6 \mu \mathrm{L}$ ) of $2 \%$ lidocaine. The third group, composed of 8 neurons, was subjected to 1 injection of $600 \mathrm{~nL} 0.25 \mathrm{M}$ GABA. We recorded a total of 24 cells in V2 during inactivation of this region.

The inactivation of all neurons produced significant effects in the majority $(80 \%)$ of cases. The statistical analysis by 2 WA showed that in slightly more than half of the cases (8/14), the cells were either directionally selective (1WA) or bidirectionally selective (2WA) and lost these properties after the GABA injections. The GABA inactivation also caused three previously non-direction selective cells to become selective to movement of the bar. Of those three, two became directionally selective (1WA) and one became bidirectionally selective (2WA).

\section{Effect on V2 neurons}

The injection of $120 \mathrm{~nL} 0.5 \mathrm{M}$ GABA in V2 caused a local decrease in the spontaneous and driven activity of V2 neurons. Before the injection, there was a high level of spontaneous activity and a strong response to the moving bar. Figure 1 illustrates a neuron from area V2 that lost its unidirectional selectivity ( $2 \mathrm{WA}, \mathrm{P}<0.05, F=56$, d.f. $=8 \times 2$ ) as a result of GABA-induced inactivation. Under the control conditions, this neuron was highly selective to movement in the direction of $315^{\circ}$. The response decreased with the injection of GABA, while the response to the direction of $180^{\circ}$ increased (1WA, $\left.\mathrm{P}>0.05\right)$. After $90 \mathrm{~min}$, the neuron again displayed selectivity toward a value quantitatively similar to initial control conditions (2WA, $\mathrm{P}<0.05)$, despite an overall lower excitability of the neuron.

Figure 2 illustrates the effect of an injection of $120 \mathrm{~nL}$ $0.5 \mathrm{M}$ GABA on the firing rate of $6 \mathrm{~V} 2$ neurons. The spontaneous activity and best direction response, in most neurons, decreased after the GABA injection, similar to what is observed following the inactivation of MT and V4. This effect is not consistent for all directions, as some neurons decreased while others increased the firing rate after GABA injection.

\section{Time course of the GABA effect}

Figure 3 illustrates the time course of the effect of an injection of $600 \mathrm{~nL} 0.25 \mathrm{M}$ GABA on the general excitability of $8 \mathrm{~V} 2$ neurons, over approximately $5 \mathrm{~h}$ of recordings. Following the GABA injection, most neurons 
A

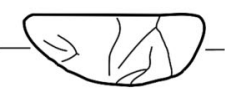

E Control

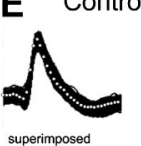

$$
135^{\circ}
$$

\section{C}

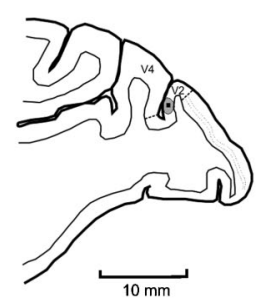

$90^{\circ} \quad 6.03$

6.03

winh

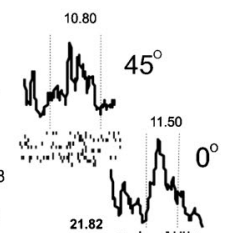

B
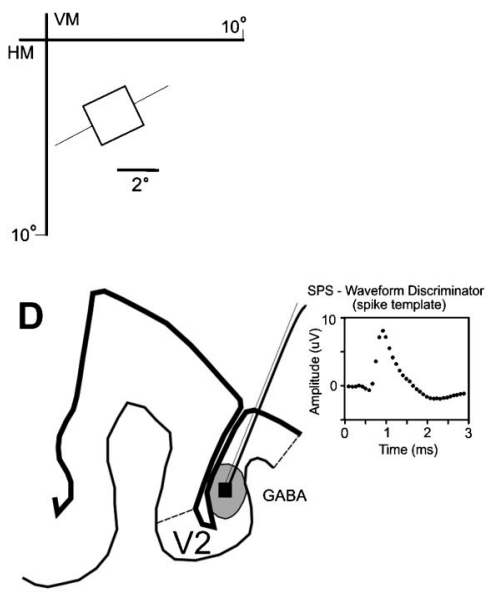

$180^{\circ}$

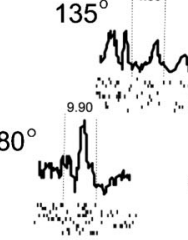

(1)

$225^{\circ}$ (M)

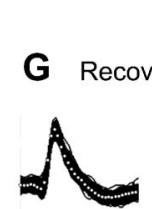

$180^{\circ}$<smiles>C1CCCCC1</smiles>

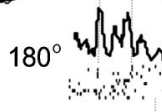

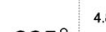

\begin{tabular}{ll|l}
$90^{\circ}$ & 3.93 \\
\hline
\end{tabular}

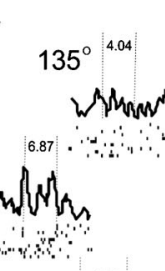

Noment

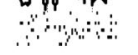

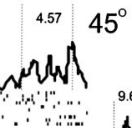

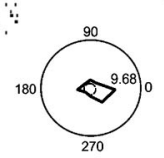

$\left.225^{\circ}\right)$ 4.86

$\because y+$<smiles>[C]1C#CC1</smiles>

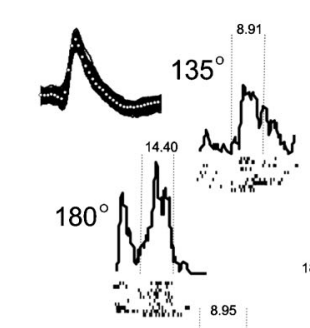

F 1 min after GABA

${ }_{5.66} 90^{\circ}$

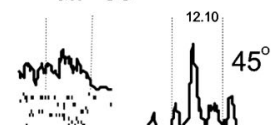

i' ....'s

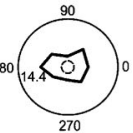

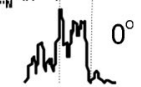

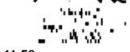

$225^{\circ}$ NM

6.63 
A

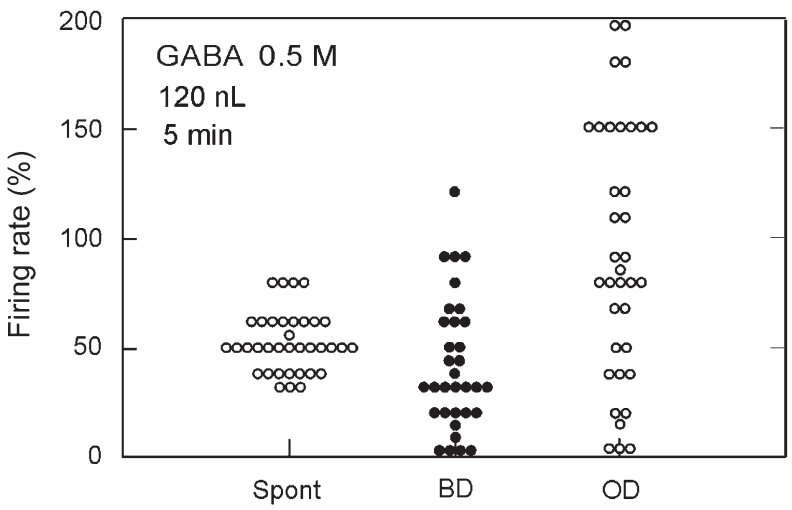

B

Spontaneous activity
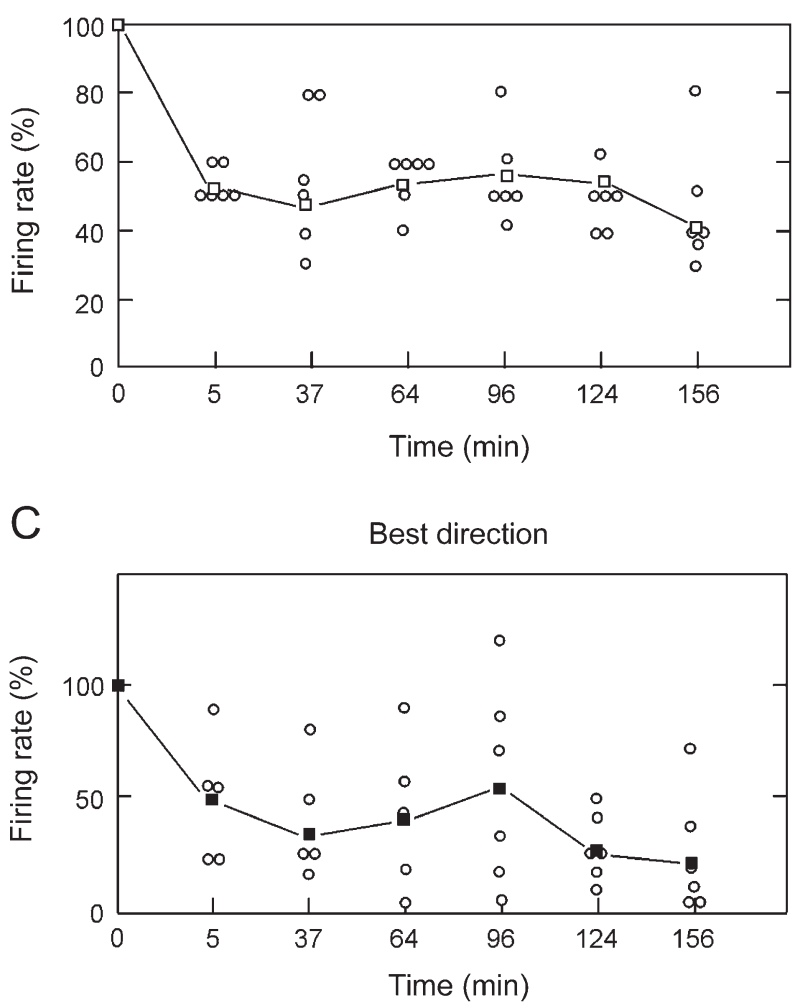

Figure 2. Strength and time course of the effect of a small GABA injection (120 nL, $0.5 \mathrm{M})$ on 6 neurons recorded in V2 during inactivation. $A$, Effect of GABA inactivation on the electrical activity of neurons shown as a percentage, normalized relative to control neurons for the spontaneous activity of neurons (Spont), best direction (BD) and other direction (OD). $B$, Time course of the effect of GABA inactivation on spontaneous activity and $C$, on the best direction of neurons over a period of 156 min. Open circles are normalized percentage of firing rate of the spontaneous activity $(B)$ and of the best response $(C)$ of each of the six neurons. Squares are the mean of normalized firing rate of the spontaneous activity $(B)$ and the response to the best direction $(C)$.

\section{Lidocaine effect}

In the second group, neurons received $2 \%$ lidocaine. Figure 4 illustrates the response of V2 neurons that lost direction selectivity as a result of lidocaine-induced inactivation (1WA, $\mathrm{P}<0.05, F=41$, d.f.=8). This cell exhibited directional selectivity during the control condition and lost its selectivity $1 \mathrm{~min}$ after lidocaine injection $(P=0.7)$. After $30 \mathrm{~min}$, the cell recovered its directional selectivity (1WA, $P=0.05, F=38$, d.f. $=8$ ). Following this injection, the response to the best orientation or direction of V2 neurons decreased by approximately $50 \%$ in 1-10 min (Figure 5). The median response rate illustrates 

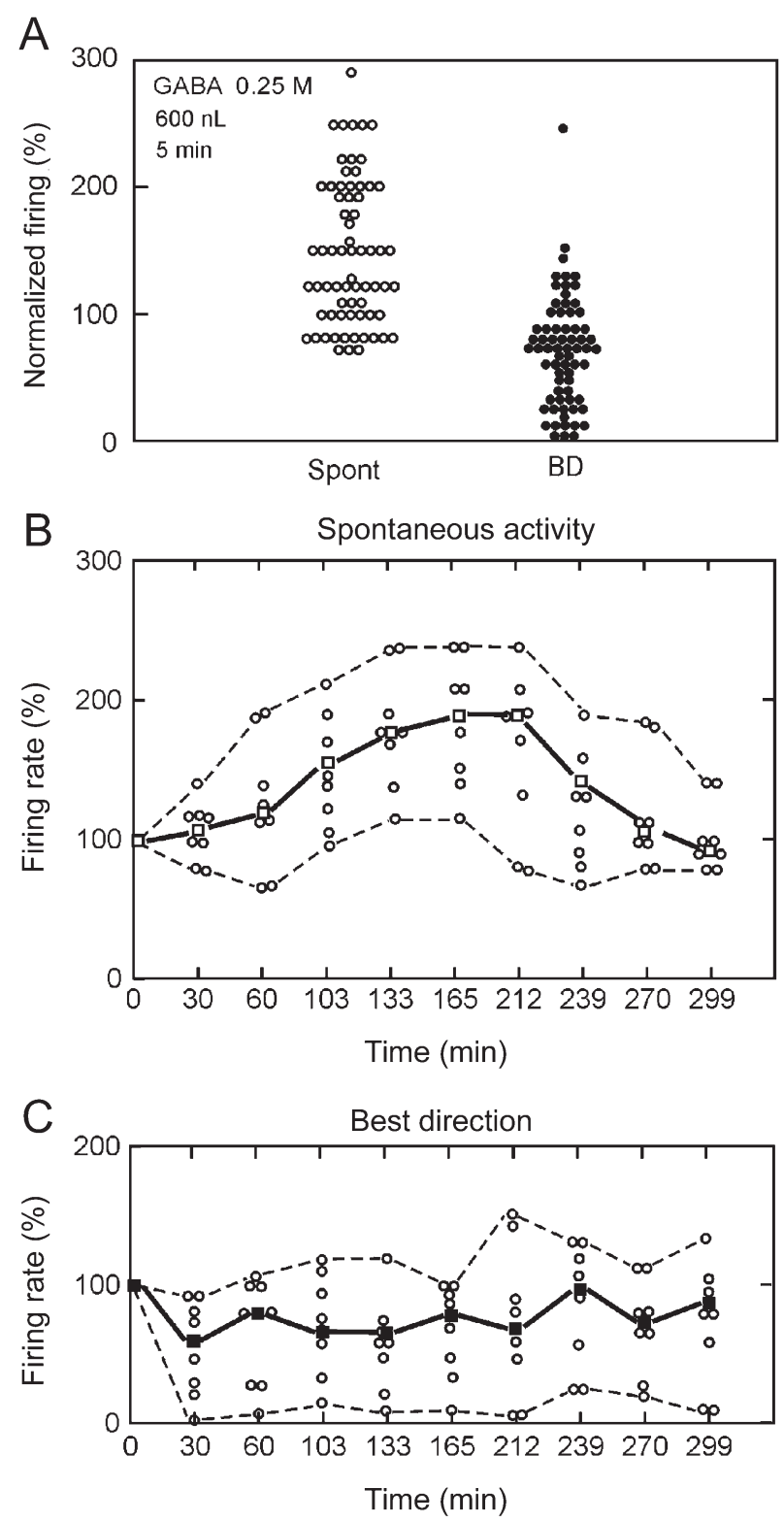

Figure 3. $A$, Strength and time course of the effect of a medium size GABA injection ( $600 \mathrm{~nL}, 0.25 \mathrm{M})$ on $B$, spontaneous activity (Spont) and $C$, best direction (BD) of 8 neurons recorded in $\mathrm{V} 2$. The relative firing rate of the cells is plotted over a period of 300 min $(9$ repeated measurements). Open circles are normalized percentage of firing rate of the spontaneous activity $(B)$ and of the best response $(C)$ of each of the six neurons. Squares are the mean of normalized firing rate of the spontaneous activity $(B)$ and the response to the best direction $(C)$.

that for both the best direction and the spontaneous activity, the trend is toward the return of the neuronal cell firing to control levels. The main effect of lidocaine injection was a reduced response to the best direction or orientation. In other directions or orientations, the result varied.

In most cases, lidocaine increased the amount of spontaneous activity (Figure $5 \mathrm{~A}$ ). Figure $5 \mathrm{~B}$ and $\mathrm{C}$ illustrate the effect of lidocaine in two neurons over approximately $30 \mathrm{~min}$ of recording. Figure 5D illustrates the initial decrease in responding, and the subsequent return toward baseline, of a V2 neuron to visual stimuli after an injection of lidocaine. Under control conditions, this neuron displayed a peak response of 20 spikes/s with its size receptive field of $4.5^{\circ}$. After the first injection of lidocaine at $13 \mathrm{~min}$, the response dropped and the signal/ noise ratio of the neuron decreased. All spontaneous activity was suppressed in the first minute after the second injection of lidocaine, which led to an improved 
A

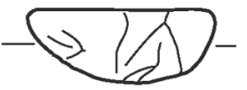

E Control

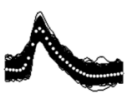

C
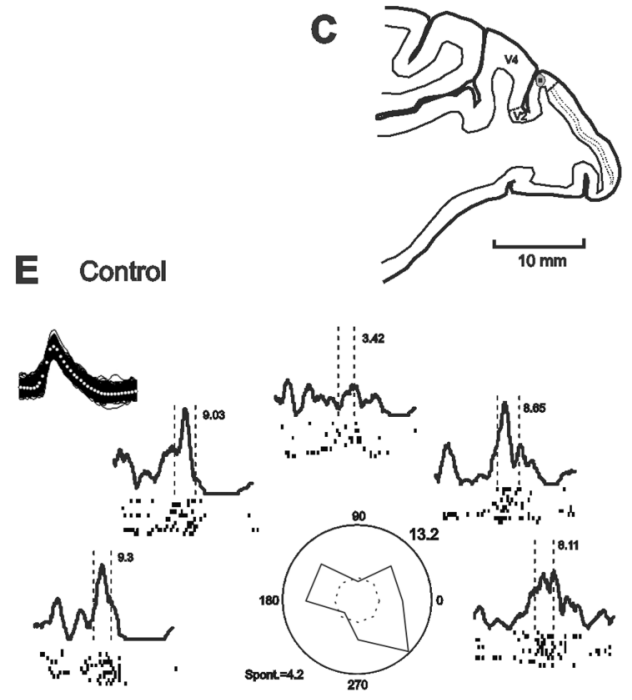

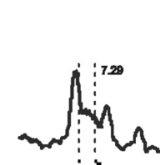

:-...4.

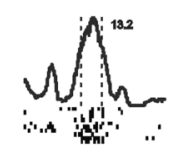

and
B

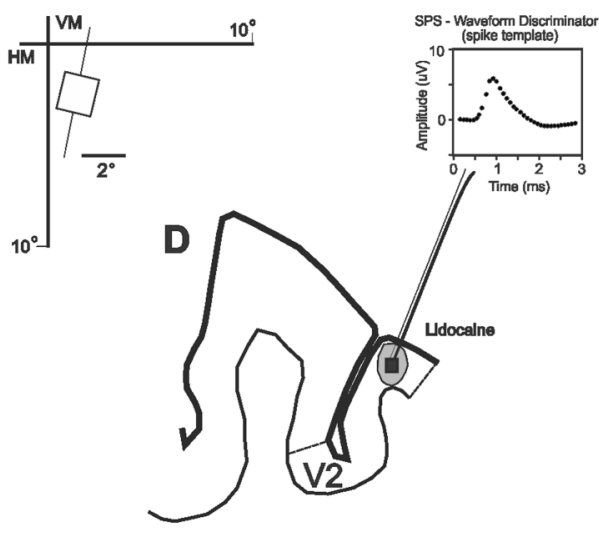

F (1 min after lidocaine)

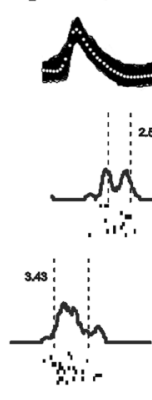

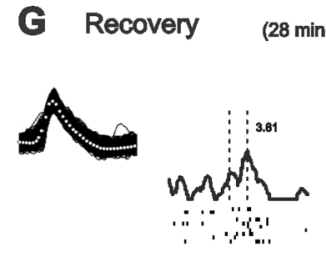

(28 min after lidocaine)

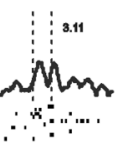

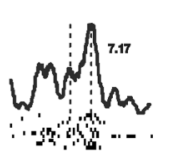<smiles></smiles>

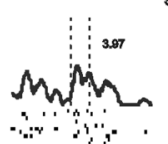

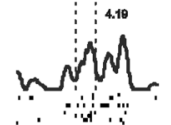

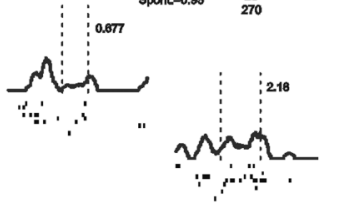

Figure 4. Effect of a large $2 \%$ lidocaine injection $(6 \mu \mathrm{L})$ on the intrinsic circuit of $\mathrm{V} 2$. A, Dorsal reconstruction of the Cebus brain, indicating the levels of the section illustrated in $C$. B, Visual field location of the receptive fields of V2 that were studied in this experiment. The small square in B corresponds to the V2 receptive field. HM: horizontal meridian: VM: vertical meridian. D, Enlarged portion of the parasagittal sections showing the location of inactivation and of the recording site in V2 (inset: SPS-8701: waveform discriminator spike template). $E$, The polargrams illustrate the $\mathrm{V} 2$ neuron mean firing rate elicited by bars moving in 8 directions orthogonal to the axis of movement (control condition, polargram at the top). Left inset: superimposed spike waveforms (black solid lines) and spike template (white dots). Spike density function and 10 trials spike rasters are illustrated for each direction. $F$, One minute after lidocaine inactivation. The loss of unidirection selectivity of a V2 neuron after a $2 \%$ lidocaine inactivation of area V2 ( $P<0.05$, oneway ANOVA). G, Twenty-eight minutes after lidocaine inactivation. 

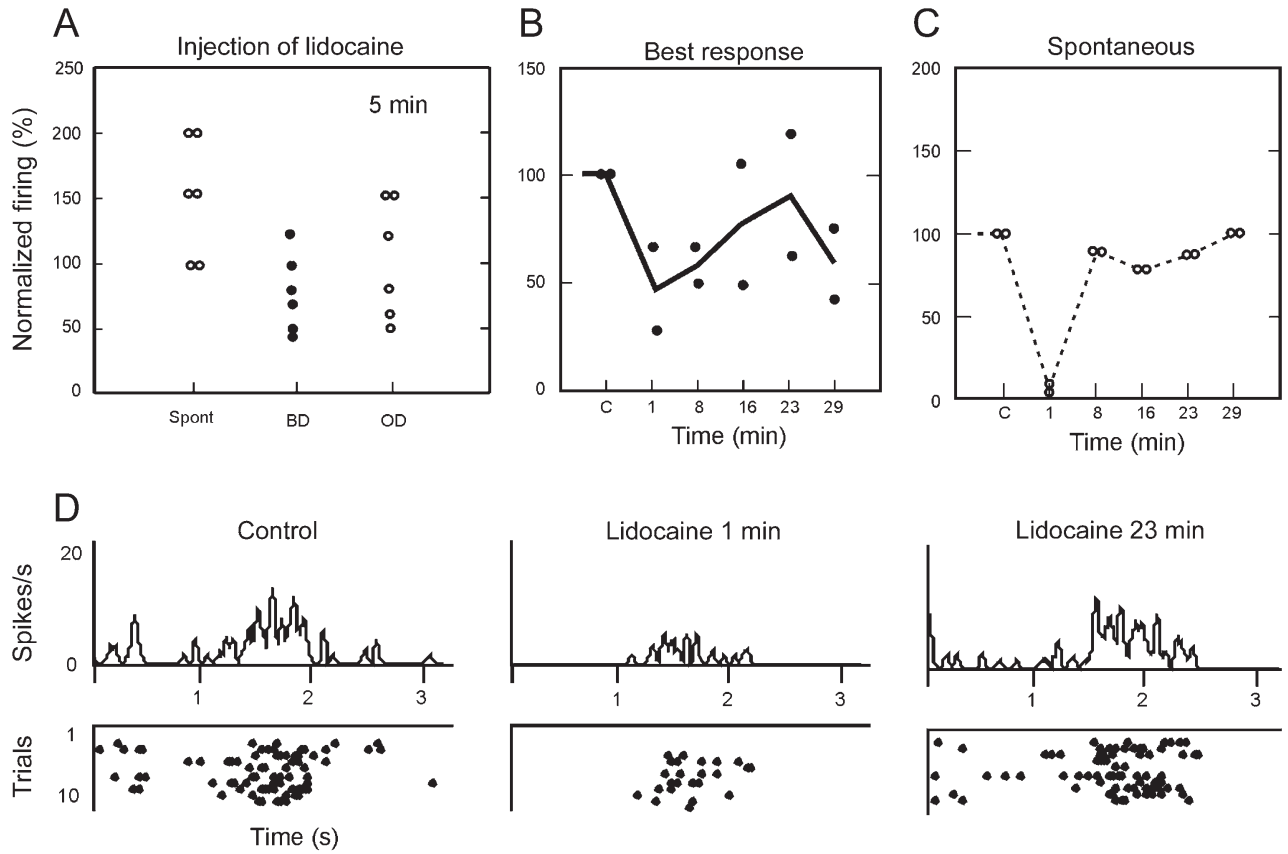

Figure 5. Effects and time course of an injection of $2 \%$ lidocaine into area $\mathrm{V} 2$ on the response of 6 neurons. $A$, Effect on the normalized firing rate for the spontaneous activity (Spont), best direction (BD) and other direction (OD) after injection. $B$, Time course of the effect of lidocaine injection on the best response and $C$, on the spontaneous activity of 6 neurons. $D$, Post-stimulus time histograms and spike raster of a neuron at three different times: before (control), and after (1 and $23 \mathrm{~min})$ the injection.

signal/noise ratio. The response recovery over time was equivalent to that of the control 23 min after injection. Thus, the effect of lidocaine appears to have three phases: the general inhibition of the response and spontaneous activity, the increase in the signal/noise ratio, and the return to control response level.
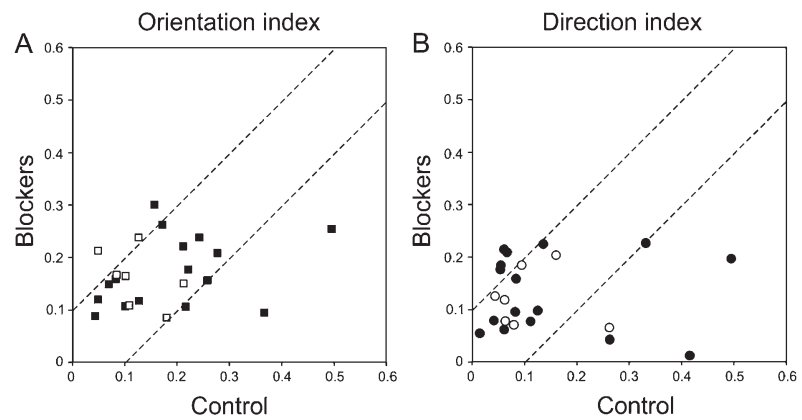

Figure 6. Direction and orientation indices of $24 \mathrm{~V} 2$ neurons. $A$, Correlation between the orientation indices before and after GABA (filled symbols) or lidocaine (open symbols) injections into area V2. $B$, Correlation between the directional indices before and after GABA or lidocaine injections. Broken lines with a slope of 1 represent the limits of significance for the changes in the indices (>0.2). Significant changes in direction index (9/24) and in orientation index $(5 / 24)$ were observed for these cells $(P<0.05$, $\chi^{2}$ test). In addition, the injection of blockers in V2 tended to decrease the orientation and direction selectivity of the neurons examined
Figure 6 shows changes in the direction and orientation tuning of 24 V2 cells after GABA- and lidocaineinduced inactivation of the area V2. When changes greater than 0.2 were used as a criterion, 14 neurons $(29.2 \%)$ changed their direction or orientation tuning.

There was no trend in the sample of cells. Direction tuning of V2 neurons either increased (4/24) or decreased $(5 / 24)$ after injections of GABA or lidocaine. In addition, these cells decreased (2/24) and increased (3/24) their orientation selectivity. The effect of GABA inactivation of V2, unlike the effect produced by inactivation of MT and V4 areas, decreases the indices of orientation or direction selectivity.

\section{Discussion}

A number of previous studies have described the intrinsic connections in area V2 in the monkey and cat $(6,7,10-13,30)$. Hupé et al. (15) performed a quantitative study on the spread of GABA after pressure injection into the cortex. An injected volume of $13 \mathrm{~nL} 0.1 \mathrm{M}$ GABA would occupy an extracellular volume of approximately $60 \mathrm{~nL}$. For injected volumes greater than $60 \mathrm{~nL}$, it would theoretically be difficult to predict the shape of the spreading. Hupé et al. (15) also determined that, when larger volumes of GABA were injected, the inactivation period and the time needed for the neurons to recover would also be greater. Our observations are in agreement 
with Hupé et al. (15) and their later results (31). In Hupé et al. (31), the neurons required $40 \mathrm{~min}$ to recover to baseline after a $0.9 \mu \mathrm{L}$ injection of $0.1 \mathrm{M}$ GABA. The duration of the inhibition described in the present study is consistent with that reported by Hupé et al. (31).

\section{Inactivation techniques}

Lidocaine, a fast action blocker, has an immediate effect that lasts less than 30 min $(32,33)$. This anesthetic works by blocking the sodium channels of the body and the axon of the neuron, thus preventing the initiation and conduct of the neural signal. The main disadvantage appears to be the inactivation of passage fibers.

The evidence demonstrating that GABA may be an inhibitory neurotransmitter in the visual cortex is attributed to Sillito (34). More recently microiontophoresis and pressure injections have been used to study the effects produced by inactivation of GABA on the line orientation and direction in areas 17 and 18 of cat and monkey $(6,7,10-13,30) . G A B A_{A}$ receptors are located mainly in the cell body and act through activation of chloride channels. These receptors are selectively blocked by bicuculline, while the chloride channel itself is blocked by picrotoxin. GABA can be applied numerous times, produce inactivation of short and long duration (26-28), and does not affect fibers of passage. Its effect depends on the mode of application (pressure or iontophoresis) producing inactivation within a distance of 2-4 $\mathrm{mm}$ (31).

Injections of lidocaine should produce an effect on the neurons of area V2 circuitry that is more intense and more widespread given that all neurons have the sodium channel. Injections of GABA should affect a specific neural population: those with $\mathrm{GABA}_{\mathrm{A}}$ receptors and thus produce a less intense and more focal effect. This theoretical prediction is consistent with the results obtained in this study. However, the optimal experimental design to answer that question definitively would be the sequential injection of two blockers in the same experiment, which would otherwise have other impeding issues.

\section{Evaluation of the early and late effects of GABA inactivation}

GABA inactivation of $V 2$ intrinsic circuitry produced early (up to $20 \mathrm{~min}$ ) and late (20-150 min) after-injection effects on intrinsic V2 neurons. The early effects consisted of a general decrease in neuronal excitability, which corresponded to a depression in the spontaneous and driven activities. The later effects generally reflected changes in the orientation and/or direction selectivity of the $\mathrm{V} 2$ neurons.

The V2-driven activity typically fell below $50 \%$ of the control level during the first $10 \mathrm{~min}$ after injection. The different effects of GABA inactivation on the spontaneous and stimulus-driven activity were complex, and we attributed them to the properties of the GABAergic channels. This dissociation suggests that different and possibly independent mechanisms underlie the changes in the stimulus-driven and spontaneous activity in the cortex.

\section{Duration of inactivation}

Prior to the start of these experiments, we were unable to predict the long-lasting effects of GABA injection into area $\mathrm{V} 2$. We believe that the inhibitory effects detected in the present study are equivalent to the effects of iontophoretic injections of GABA described in the literature $(7,11,12)$. With iontophoresis, however, the amount of GABA released is usually much smaller than that released from a pressure injection. It is therefore possible that, in addition to an initial inactivation effect, a pressure injection causes late effects in neuronal activities that are not observed when lower quantities of GABA are injected. Several changes occurred in the V2 neurons, suggesting that V2 neurons could be influenced by GABA inactivation. These effects persisted for 40-299 min.

The effects of pharmacological inactivation are usually reversible and are evaluated in comparison to the preinjection state. A return to the resting or pre-injection spontaneous and driven activity is indicative of a non-toxic or non-destructive effect on the central nervous system tissue. Therefore, we based our results on a sample of cells in which a clear trend towards the recovery of the driven activity toward baseline was observed.

\section{V2 cortical circuits}

Several studies have demonstrated the role of intrinsic circuits in the regulation of receptive field properties $(6,7,10-13,30)$. The intrinsic connectivity of V2 was studied by Rockland (35) in S. sciureus and Macaca spp. The connections of area $\mathrm{V} 2$ originate from pyramidal neurons in layers III and V. The extent of the tangential connections from V2 varied from 2.5 to $3.0 \mathrm{~mm}$ (36). In Saimiri sciureus the areas of orientation in V2 formed granules; the size and distance between these granules were double those in V1 (37). In V2, in Macaca fascicularis (38) the results found were very similar to those of $S$. sciureus (37). In the present study, we provide evidence that intrinsic horizontal and vertical connectivity from area V2 is capable of not only modulating the spontaneous and driven activity of V2 neurons but also modifying $\mathrm{V} 2$ receptive field properties, including orientation and direction selectivity.

We propose the following hypothetical inactivation circuits to explain the results described in the present study. The intrinsic circuits modify the properties of the receptive field, probably through excitatory and inhibitory neurons. The most common effect observed in the first 5-10 min of GABA injection in V2 was a decrease in both spontaneous and driven activity of V2 neurons. We propose that pyramidal neurons that modulate directional selectivity in V2 contain $\mathrm{GABA}_{\mathrm{A}}$ receptors (6) that capture the injected GABA and lead to a more negative membrane potential. This change results in an inhibition of the 
propagation of activity in the neurons that project to both the superficial and deep layers of V2. There is also a decrease in the release of the neurotransmitters from the neurons of area V2. As a result, the excitatory synapses decrease, causing a decrease in spontaneous and driven activity of the V2 neurons. The injections affect all direction selectivity columns, resulting in a decrease of spontaneous and driven activity of the neurons in all directions.

A loss of selectivity was the most frequently detected receptive field alteration in the V2 neurons after GABA inactivation. We hypothesize the existence of a circuit involving a projection of neurons (likely pyramidal neurons) from deep and superficial layers of area V2 containing $\mathrm{GABA}_{A}$ receptors. The excitability of these neurons would decrease after the activation of $G_{A B A}$ receptors. This decrease in excitability would influence the pyramidal neurons in the V2 area that receive these projections and would also influence intrinsic inhibitory neurons. Intrinsic inhibitory interneurons decrease their influence on neuronal afferents to neighboring columns and cause a loss of direction selectivity for the majority of neurons. The directionality of the remaining $10 \%$ of neurons in our population became selective after the GABA injection. Therefore, we propose that the inactivation of area V2 has partial and asymmetrical effects, which causes some direction columns to remain active while others become suppressed. This asymmetrical inhibition would generate direction selectivity in neurons that were pan-directional before the injection.

\section{Optimization of the use of the animal model}

We used one $C$. apella monkey in 5 recording sessions performed once a week to collect data from V2 neurons immediately before and after the injection of GABA. We aimed to record from as many cells as necessary in several experimental sessions in one animal

\section{References}

1. Hubel DH, Wiesel TN. Receptive fields functional architecture of monkey striate cortex. J Physiol 1968; 195: 215-243.

2. Lamme VA, Roelfsema PR. The distinct modes of vision offered by feedforward recurrent processing. Trends Neurosci 2000; 23: 571-579, doi: 10.1016/S0166-2236(00)01657-X.

3. Angelucci A, Levitt JB, Lund JS. Anatomical origins of the classical receptive field modulatory surround field of single neurons in macaque visual cortical area V1. Prog Brain Res 2002; 136: 373-388, doi: 10.1016/S0079-6123(02)36031-X.

4. Douglas RJ, Martin KA. A functional microcircuit for cat visual cortex. J Physiol 1991; 440: 735-769.

5. Gilbert CD, Wiesel TN. Columnar specificity of intrinsic horizontal corticocortical connections in cat visual cortex. $J$ Neurosci 1989; 9: 2432-2442.

6. Sato $\mathrm{H}$, Katsuyama $\mathrm{N}$, Tamura $\mathrm{H}$, Hata $\mathrm{Y}$, Tsumoto $\mathrm{T}$. Mechanisms underlying orientation selectivity of neurons in the primary visual cortex of the macaque J Physiol 1996; 494: 757-771. to have a statistically significant sample. The use of a single animal is justified to preserve as many primates as possible. Other studies that used only one animal but had statistically significant samples have been previously published $(39,40)$.

\section{GABA inactivation on the intrinsic, feed-forward and feedback circuits}

We should consider the effect of GABA inactivation on the feed-forward, intrinsic and feedback circuits. There are four possible targets for the GABA injections in V2. The first is a direct effect on the recorded neuron. The second is an indirect effect involving an intrinsic V2 neuron that projects to the recorded neuron. The third is an indirect effect involving feed-forward projections, and the fourth is an indirect effect involving feedback projections. The results obtained with small injections of GABA on the orientation and direction selectivity of $\mathrm{V} 2$ neurons are different from the results obtained with injections of GABA in the feedback loop of V2. While injections in V4 or MT increase the orientation and direction indices, direct injections in V2 decrease the orientation and direction indices. The results suggest that small amounts of GABA interfere only with intrinsic neurons that receive axonal terminals from feedback circuits.

The large injections of lidocaine produced a robust inhibitory effect on the activity of the V2 cells and they were used as control for the inhibitory component of GABA injections.

\section{Acknowledgments}

Research supported by PRONEX, CNPq, FUJB, FAPERJ, FINEP, and CEPG. A.K. Jansen-Amorim received $C N P q$ fellowships for her graduate studies. $R$. Gattass is the recipient of a CNPq research fellowship.

7. Crook JM, Kisvarday ZF, Eysel UT. Evidence for a contribution of lateral inhibition to orientation tuning direction selectivity in cat visual cortex: reversible inactivation of functionally characterized sites combined with neuroanatomical tracing techniques. Eur J Neurosci 1998; 10: 20562075, doi: 10.1046/j.1460-9568.1998.00218.x.

8. Sillito AM. The contribution of inhibitory mechanisms to the receptive field properties of neurones in the striate cortex of the cat. J Physiol 1975; 250: 305-329.

9. Sillito AM. The effectiveness of bicuculline as an antagonist of GABA visually evoked inhibition in the cat's striate cortex. J Physiol 1975; 250: 287-304.

10. Crook JM, Eysel UT. GABA-induced inactivation of functionally characterized sites in cat visual cortex (area 18): effects on orientation tuning. J Neurosci 1992; 12: 1816-1825.

11. Crook JM, Kisvarday ZF, Eysel UT. GABA-induced inactivation of functionally characterized sites in cat visual cortex 
(area 18): effects on direction selectivity. J Neurophysiol 1996; 75: 2071-2088.

12. Crook JM, Kisvarday ZF, Eysel UT. GABA-induced inactivation of functionally characterized sites in cat striate cortex: effects on orientation tuning direction selectivity. Vis Neurosci1997; 14:141-158, doi: 10.1017/S095252380000883X

13. Sato $H$, Katsuyama $N$, Tamura $H$, Hata $Y$, Tsumoto $T$. Mechanisms underlying direction selectivity of neurons in the primary visual cortex of the macaque. $J$ Neurophysiol 1995; 74: 1382-1394.

14. Rosa MG, Sousa AP, Gattass R. Representation of the visual field in the second visual area in the Cebus monkey. J Comp Neurol 1988; 275: 326-345, doi: 10.1002/cne.902750303.

15. Hupé JM, Chouvet G, Bullier J. Spatial temporal parameters of cortical inactivation by GABA. J Neurosci Methods 1999; 86: 129-143, doi: 10.1016/S0165-0270(98)00162-9.

16. Gattass R, Oswaldo-Cruz E, Sousa APB. Visuotopic organization of the Cebus pulvinar: a double representation the contralateral hemifield. Brain Res 1978; 152: 1-16, doi: 10.1016/0006-8993(78)90130-0.

17. Gattass R, Sousa APB, Oswaldo-Cruz E. Single unit response types in the pulvinar of the Cebus monkey to multisensory stimulation. Brain Res 1978; 158: 75-87, doi: 10.1016/0006-8993(78)90007-0.

18. Gattass R, Oswaldo-Cruz E, Sousa APB. Visual receptive fields of units in the pulvinar of Cebus monkey. Brain Res 1979; 160: 413-430, doi: 10.1016/0006-8993(79)91070-9.

19. Amorim AKJ, Picanco-Diniz CW. Morphometric analysis of intrinsic axon terminals of Cebus monkey area 17. Braz $J$ Med Biol Res 1996; 29: 1363-1368.

20. Amorim AKJ, Picanco-Diniz CW. Intrinsic projections of Cebus-monkey area 17: cell morphology axon terminals. Rev Bras Biol 1996; 56 (Suppl 1): 209-219.

21. Amorim AKJ, Picanco-Diniz CW. Horizontal projections of area 17 in Cebus monkeys: metric features modular laminar distribution. Braz J Med Biol Res 1997; 30: 1489-1501, doi: 10.1590/S0100-879X1997001200018.

22. Gattass R, Sousa APB, Rosa MG. Visual topography of V1 in the Cebus monkey. J Comp Neurol 1987; 259: 529-548, doi: 10.1002/cne.902590404.

23. Fiorani M, Gattass R, Rosa MG, Sousa APB. Visual area MT in the Cebus monkey: location visuotopic organization variability. J Comp Neurol 1989; 287: 98-118, doi: 10.1002/ cne.902870108.

24. Piñon MC, Gattass R, Sousa APB. Area V4 in Cebus monkey: extent visuotopic organization. Cereb Cortex 1998; 8: 685-701, doi: 10.1093/cercor/8.8.685.

25. Gattass R, Nascimento-Silva S, Soares JG, Lima B, Jansen $A K$, Diogo AC, et al. Cortical visual areas in monkeys: location, topography, connections, columns, plasticity cortical dynamics. Philos Trans R Soc Lond B Biol Sci 2005; 360: 709-731, doi: 10.1098/rstb.2005.1629.

26. Jansen-Amorim AK, Lima B, Fiorani M, Gattass R. GABA inactivation of visual area MT modifies the responsiveness direction selectivity of V2 neurons in Cebus monkeys. Vis Neurosci 2011; 28: 513-527, doi: 10.1017/ S0952523811000411

27. Jansen-Amorim AK, Fiorani M, Gattass R. GABA inactivation of area V4 changes receptive-field properties of V2 neurons in Cebus monkeys. Exp Neurol 2012; 235: 553562, doi: 10.1016/j.expneurol.2012.03.008.

28. Soares JG, Diogo AC, Fiorani M, Souza APB, Gattass R. Effects of inactivation of the lateral pulvinar on response properties of second visual area cells in Cebus monkeys. Clin Exp Pharmacol Physiol 2004; 31:580-590, doi: 10.1111/j.1440-1681.2004.04051.x.

29. Wang C, Waleszczyk WJ, Burke W, Dreher B. Modulatory influence of feedback projections from area 21a on neuronal activities in striate cortex of the cat. Cereb Cortex 2000; 10: 1217-1232, doi: 10.1093/cercor/10.12.1217.

30. Kisvarday ZF, Crook JM, Buzas P, Eysel UT. Combined physiological-anatomical approaches to study lateral inhibition. J Neurosci Methods 2000; 103: 91-106, doi: 10.1016/ S0165-0270(00)00299-5.

31. Hupé JM, James AC, Girard P, Bullier J. Response modulations by static texture surround in area V1 of the macaque monkey do not depend on feedback connections from V2. J Neurophysiol 2001; 85: 146-163.

32. Martin JH, Cooper SE, Ghez C. Differential effects of local inactivation within motor cortex red nucleus on perfomance of an elbow task in the cat. Exp Brain Res 1993; 94: 418-428.

33. Maunsell JHR, Nealey TA, DePriest DD. Magnocellular parvocellular contributions to responses in the middle temporal visual area (MT) of the macaque monkey. $J$ Neurosci 1990; 10: 3323-3334.

34. Sillito AM. Proceedings: Modification of the receptive field properties of neurones in the visual cortex by bicuculline, a GABA antagonist. J Physiol 1974; 239: 36P-37P.

35. Rockland KS. A reticular pattern of intrinsic connections in primate area V2 (area 18). J Comp Neurol 1985; 235: 467478, doi: $10.1002 /$ cne. 902350405 .

36. Livingstone MS, Hubel DH. Specificity of intrinsic connections in primate primary visual cortex. $J$ Neurosci 1984; 4 2830-2835.

37. Malach R, Tootell RB, Malonek D. Relationship between orientation domains, cytochrome oxidase stripes, and intrinsic horizontal connections in squirrel monkey area V2. Cereb Cortex 1994; 4: 151-165, doi: 10.1093/cercor/4.2.151.

38. Levitt JB, Yoshioka T, Lund JS. Intrinsic cortical connections in macaque visual area V2: evidence for interaction between different functional streams. J Comp Neurol 1994; 342: 551-570, doi: 10.1002/cne.903420405.

39. Lima B, Fiorani M, Gattass R. Modulation by context of a scene in monkey anterior inferotemporal cortex during a saccadic eye movement task. An Acad Bras Cienc 2003; 75: 71-76, doi: 10.1590/S0001-37652003000100008.

40. Lima B, Fiorani M, Gattass R. Changes of ongoing activity in Cebus monkey perirhinal cortex correlate with behavioral performance. Braz J Med Biol Res 2005; 38:59-63. 